\title{
Bioequivalencia en medicamentos.
}

\section{Bioequivalence studies in drugs.}

Dos medicamentos son bioequivalentes si ambos alcanzan la circulación sistémica de modo similar, logrando las mismas concentraciones en la sangre, es decir que son igualmente biodisponibles y en consecuencia su eficacia y seguridad son las mismas. Un estudio de bioequivalencia es un ensayo clínico en voluntarios sanos, a los que se administra el medicamento genérico o el innovador de referencia, en ayunas, separados por un periodo de lavado de más de 3 veces el tiempo de vida media. Durante el estudio se toman muestras seriadas de sangre para construir una curva tiempo-concentración plasmática del principio activo, si ambas curvas se superponen existirá bioequivalencia. Esta metodología ha sido ampliamente utilizada en los países desarrollados como Estados Unidos (1), Canadá (2), Japón (3), y en la Unión Europea (4), siendo recomendada por la OMS (5).

Los estudios de bioequivalencia permiten afirmar que un medicamento genérico es intercambiable con el producto innovador (5). El innovador es aquel que salió primero al mercado y que recibió la autorización de comercialización por la demostración de eficacia y seguridad en base a ensayos clínicos.

$\mathrm{Al}$ otorgar una patente a un medicamento innovador se le permite una exclusividad en el mercado por 20 años. Cuando la patente ha expirado, diversas compañías que elaboran productos genéricos pueden empezar a producir medicamentos con el mismo principio activo, a este tipo de medicamento se conoce como medicamento de múltiples fuentes (genérico o llamados también "similares").

Pero hay situaciones en la cuales no se exige formalmente la bioequivalencia (6); esto se da por ejemplo si la aplicación es por vía intravenosa, ya que al no haber disolución ni absorción, la biodisponibilidad es la misma; en esta circunstancia el medicamento genérico se comporta de modo similar al innovador. Se reconoce que no se debe exigir pruebas de bioequivalencia, cuando se traten de soluciones acuosas de uso parenteral, soluciones orales exentas de excipientes conocidos que modifiquen los parámetros farmacocinéticos, gases inhalatorios, medicamentos tópicos de uso no sistémico y cuya absorción no represente riesgo, medicamentos para inhalación en solución acuosa, y medicamentos para inhalación en suspensión y cuyo tamaño de partícula sea demostradamente igual al del innovador. Esto significa que estos tipos de medicamentos no requieren de pruebas de bioequivalencia.

El conocimiento de las características de la disolución acuosa y de la absorción intestinal de los fármacos que tienen forma farmacéutica sólida oral de liberación inmediata, se utiliza como base para un Sistema de Clasificación Biofarmaceutica (7), que permite agrupar a los principios activos en 4 clases: la Clase I de alta solubilidad y alta permeabilidad, la Clase II de baja solubilidad y alta permeabilidad, la Clase III de alta solubilidad y baja permeabilidad, y la Clase IV de baja solubilidad y baja permeabilidad. A partir de ésta clasificación, se establece que en algunos medicamentos, la bioequivalencia convencional, pueda ser remplazada por la bioequivalencia in vitro, permitiendo una exención de la necesidad de bioequivalencia in vivo, que requiere realizarse en humanos.

Los estudios de bioequivalencia in vitro están constituidos por estudios comparativos de perfiles de disolución, en donde se determina la cantidad o porcentaje del principio activo disuelto en función del tiempo bajo condiciones controladas y validadas. Para productos farmacéuticos altamente solubles, y altamente permeables, la bioequivalencia in vitro (estudios de disolución) es apropiada y considerada como criterio necesario y suficiente para comparar el medicamento innovador y el de fuentes múltiples. El uso de estas técnicas ha permitido no exigir bioequivalencia in vivo para un número importante de medicamentos (8).

El 27 de febrero de 1985 en Estados Unidos de Norteamérica se venció la patente de Valium ${ }^{\circledR}$, que era el medicamento innovador, el propietario de la patente, Hoffmann-La Roche; dos semanas antes presentó un pedido para no admitir productos genéricos, ya que según su opinión los métodos utilizados no garantizaban la equivalencia 
(9). Sin embargo, el pedido fue desestimado y para setiembre de ese año, la FDA había concedido 3 autorizaciones para genéricos (10).

Para las tabletas de diazepam al inicio se realizaba bioequivalencia in vivo (11). Sin embargo, ahora, la FDA considera tributario de bioequivalencia in vitro (12). En el fascículo anterior de la Revista Médica Herediana se publicó un artículo sobre bioequivalencia de tabletas de Diazepam de $10 \mathrm{mg}$ (13), el resultado muestra que el genérico que se compró en la farmacia del MINSA es similar al innovador, mas no el que se compró en una botica particular. El estudio muestra la necesidad de contar con los estudios de bioequivalencia, como sostén de la intercambiabilidad de estos medicamentos.

La implementación de esta exigencia para la obtención de la autorización sanitaria para la venta, no estaba contemplada en la legislación anterior; sin embargo, con la aprobación de la Ley 29459 Ley de los productos farmacéuticos, dispositivos médicos y productos sanitarios y la nueva reglamentación, gradualmente se implementará, teniendo en cuenta el Riesgo sanitario de los medicamentos según la propuesta en la directiva de equivalencia terapéutica de la Dirección General de Medicamentos Insumos y Drogas (14).

\section{Dr. Leandro Huayanay-Falconí ${ }^{1}$}

\section{REFERENCIAS BIBLIOGRÁFICAS}

1. US Department of Health and Human Services FDA CDER. Guidance for Industry Bioavailability and Bioequivalence. Studies for Orally Adminstered Drug Products- General Considerations Rockville, MD CDER 2003. URL disponible en:http://www.fda.gov/downloadsDrugs/.../ Guidances/ucm070124.pdf (Fecha de acceso: 25 de setiembre 2012).

2. Health Canada TPD. Conduct and analysis of bioavailability and bioequivalence studies - Part A: Oral dosage formulations used for systemic effects. Ottawa Health Canada TPD; 1992. URL disponible en:. http://www.hc-sc.gc.ca/ dhp-mps/alt_formats/pdf/prodpharma/applicdemande/guide-ld/bio/gd_standards_ld_normes-eng. pdf (Fecha de acceso: 25 de setiembre 2012).

3. National Institute of Health Sciences J. Guideline for Bioequivalence Studies for Generic Products. 2212-1997. URL disponible en: http://www.nihs.go.jp/ drug/be-guide(e)/Generic/QA-E_120229_BE.pdf (Fecha de acceso: 25 de setiembre 2012).

4. Committee for Medicinal Products for Human Use (CHMP). Guideline on the Investigation of Bioequivalence. 20-1-2010. London, European Medicines Agency (EMA). URL disponible en: http:// www.emea.europa.eu/docs/en_GB/document_library/ Scientific_guideline/2010/01/WC500070039.pdf (Fecha de acceso: 25 de setiembre 2012).

5. WHO. Multisource (Generic) Pharmaceuticals Products: Guidelines On Registration Requirements to Establish Interchangeability. In WHO Expert Committee On Specifications for Pharmaceutical Preparations, Fortieth Report. Geneva, World Health Organization, 2006. Technical Report Series, No 937. Annex 7:347-390.
6. Patel J, Aneja K, Tiwari R. A review on bioavailability and bioequivalence trials and its necessity. Int $\mathrm{J}$ Pharmacy Pharm Sci. 2010; 2(3): 1-8.

7. Amidon GL, Lennemas H, Shah VP, Crison JR. A theoretical basis for a biopharmaceutic drug classification: the correlation of in vitro drug product dissolution and in vivo bioavailability. Pharmaceutics Research. 1995; 12:413-420.

8. World Health Organization. Proposal to Waive the In Vivo Bioequivalence Requirements for the Model List of Essential Medicines Immediate Release, Solid Oral Dosage Forms. In WHO Expert Committee On Specifications for Pharmaceutical Preparations, Fortieth Report. Geneva, World Health Organization, 2006. Technical Report Series, No 937. Annex 8:391437.

9. Sun M. Generics, Roche joust for Valium market. Science. 1985; 228(4698):472-3.

10. Sum M. Generic valium approved by FDA. 1985; 229(4719): 1247.

11. Locniskar A, Greenblatt DJ, Harmatz JS, Shader RI. Bioinequivalence of a generic brand of diazepam. Biopharm Drug Dispos. 1989; 10(6):597-605.

12. U.S. Food and Drug Administration. FDA Dissolution Methods.URL disponible en: http://www.accessdata. fda.gov/scripts/cder/dissolution/dsp_SearchResults Dissolutions.cfm (Fecha de acceso: 25 de setiembre 2012).

13.Herrera-Calderón O, Grande-Ortiz M. Equivalencia terapéutica de tabletas de diazepam dispensadas en la ciudad de Ica, Perú. Rev Med Hered. 2012; 23(3):154159.

14. DIGEMID. Directiva para establecer equivalencia terapéutica de medicamentos Lima: DIGEMID. URL disponible en: http://www.digemid.minsa.gob.pe/ servicios/proyecto\%20reglamento\%20equivalencia. pdf (Fecha de acceso: 25 de setiembre 2012).

1. Asesor de la Dirección General de Medicamentos, insumos y drogas (DIGEMID). Ministerio de Salud. Lima, Perú. 